\title{
Ionizing radiation activates expression of FOXO3a, Fas ligand, and Bim, and induces cell apoptosis
}

\author{
JER-YEN YANG ${ }^{1,2}$, WEIYA XIA ${ }^{1}$ and MICKEY C.-T. HU ${ }^{1,2}$ \\ ${ }^{1}$ Department of Molecular and Cellular Oncology, and ${ }^{2}$ Graduate School of Biomedical Sciences, \\ The University of Texas M.D. Anderson Cancer Center, Houston, TX 77030, USA
}

Received December 19, 2005; Accepted February 3, 2006

\begin{abstract}
Genotoxic stress such as ionizing radiation can induce DNA damage and promote cell-cycle arrest or apoptosis through either a p53-dependent or -independent pathway. Recently, members of the FOXO Forkhead transcription factor family have been implicated in playing a role in both DNA repair and apoptosis in mammalian cells that promoted us to examine the role of FOXO transcription factors in ionizing radiation-induced apoptosis. Here, we show that ionizing radiation can promote FOXO3a (FKHRL1) transcriptional activity and protein expression level, and induce nuclear translocation of FOXO3a in Saos2, a p53-null osteosarcoma cell line. Ionizing radiation stimulates expression of apoptosisinducing proteins such as Fas ligand and the Bcl-2 interacting mediator of cell death (Bim) leading to cellular apoptosis. The observed upregulation of proapoptotic genes and apoptosis in cells without p53 in response to ionizing radiation suggests a novel p53-independent mechanism underlying ionizing radiation-induced apoptosis in cancer cells.
\end{abstract}

\section{Introduction}

Genotoxic stress such as radiation in mammalian cells can induce genetic alterations, genomic instability, chromosomal rearrangement, cell transformation and tumorigenesis, or cellular apoptosis $(1,2)$. Genotoxic stress is a primary cause of DNA damage that leads to DNA repair or cell-cycle arrest or apoptosis through either a p53-dependent or -independent pathway (3-5). In the presence of $\mathrm{p} 53$, genotoxic stress can activate p53 function and result in cell-cycle arrest or cellular apoptosis. However, in the absence of p53, genotoxic stress can also induce cell-cycle arrest or apoptosis through a complicated mechanism that remains largely unclear at present. For instance,

Correspondence to: Dr M. Hu, Department of Molecular and Cellular Oncology, The University of Texas M.D. Anderson Cancer Center, Houston, TX 77030, USA

E-mail: michu@mdanderson.org

Key words: apoptosis, FOXO, Forkhead, Bim, Fas ligand, p53, radiation, tumor suppression it is unknown why some cells trigger cell cycle arrest and others become apoptotic when they are under genotoxic stress, probably depending on the physiological conditions and cell types, and the mechanism that confers the cell-type specificity remains to be elucidated. Interestingly, it has been recently shown that loss of the function of the Forkhead FOXO (Forkhead box, class $\mathrm{O}$ ) transcription factors in cancer cells may impair or decrease their ability to promote cell-cycle arrest or apoptosis when the cells are suffering from DNA damage or genomic instability due to genotoxic stress, thereby leading to tumor development (6-12).

In the presence of survival signal stimulation, Akt regulates gene expression through modulating the activity of the FOXO factors, FOXO1 (also known as FKHR), FOXO3a (FKHRL1), and FOXO4 (AFX), by phosphorylating them at three conserved serine/threonine residues (e.g. $\mathrm{Thr}_{32}, \mathrm{Ser}_{253}$, and $\mathrm{Ser}_{315}$ of FOXO3a) $(6,7)$. This phosphorylation leads to the release of the FOXO transcription factors from the DNA and translocation of those factors to the cytoplasm, where 14-3-3 protein binds to the phosphorylated FOXO factors and retains them in the cytoplasm, presumably by masking their nuclear localization signal and preventing their return to the nucleus. However, in the absence of stimulation by growth factors or survival signals, Akt [also known as protein kinase B (PKB)] is inactivated in quiescent cells, which results in retention of FOXO factors in the nucleus and upregulation of the expression of specific target genes that modulate the metabolic state $(6,13)$, control cell cycle progression such as the cyclin-dependent kinase inhibitor p27 kip1 (14) and Rb2 (p130) $(15,16)$, regulate the mitotic program such as cyclin B and Polo-like kinase (17), or induce cellular apoptosis through upregulation of Fas ligand (Fas-L) (18), the Bcl-2 interacting mediator of cell death (Bim) $(19,20)$, and TRAIL $(21,22)$. Therefore, the negative regulation of FOXO factors may have a pivotal role in controlling cell cycling or cell survival and thus may contribute to tumorigenesis. In addition to Akt, we have recently found that I $\mathrm{B}$ kinase (IKK)- $\beta$ plays a key role in regulating FOXO3a function and established a new mechanism for an Akt-independent repression of $\mathrm{FOXO} 3 \mathrm{a}$ that promotes cell proliferation and tumorigenesis (23). Moreover, we found that the level of nuclear FOXO3a in human primary breast tumor specimens correlates inversely with expression of IKK 3 in those specimens and positively with the survival rate in breast cancer, suggesting that FOXO3a is a critical tumor suppressor in human breast cancer and perhaps other types of cancer (23). 
The FOXO protein has been reported to regulate proapoptotic genes such as Fas-L and Bim transcriptionally upon cytokine withdrawal $(7,19,20)$; Bim expression appears to be restricted to certain cell types derived from the hematopoietic lineage. Strikingly, recent studies have shown that FOXO3a plays a central role in mediating ultraviolet (UV) irradiation-induction of the promoter of growth arrest and DNA damage protein 45 (GADD45A) (24). Thus, we have examined the potential role of FOXO transcription factors in radiation-induced cellular response. Our results show that FOXO3a is upregulated and translocated into the nucleus and its target genes, Fas-L and Bim, are activated in response to ionizing radiation (IR) or UV irradiation, suggesting that FOXO3a may play an important role in apoptosis in response to genotoxic stress. This phenomenon is detected in Saos2 cells, which are p53-null, and likely in MDA-MB-468 cells, which are p53-mutant; therefore, the observed upregulation of FOXO3a and Bim and cell apoptosis by radiation may be through a p53-independent pathway. Based on our findings, we propose that FOXO family members may upregulate proapoptotic genes that contribute to the cellular apoptosis response to IR or UV irradiation in a p53-independent manner.

\section{Materials and methods}

Plasmids and antibodies. Plasmids of wild-type human FOXO3a (pBS-FKHRL1) and FRE-Luc [a luciferase (Luc) reporter gene is driven by a TK promoter containing multiple copies of the FOXO-responsive elements (FRE)] were kindly provided by K.C. Arden (University of California, San Diego, CA) (25). The FOXO3a mammalian expression vector was constructed by subcloning of the full-length FOXO3a cDNA fragment containing the haemagglutinin (HA)-tag at the aminoterminus using the PCR technique (23), and the sequences were confirmed by automated DNA sequencing at our DNA Core facility. The human Fas-L 5'-flanking plasmid was provided by C.V. Paya (Mayo Clinic, Rochester, Minnesota, MI), and the Fas-L-Luc vector was constructed by subcloning a 955-bp (nucleotide locations between -956 and -2) fragment containing Fas-L promoter-enhancer region into a Luc reporter pGL2 Basic vector (Promega) upstream of the Luc gene. The DNA dye, DAPI, was obtained from Roche. The Dual-Luciferase Reporter assay system was purchased from Promega. A polyclonal antibody against FOXO3a (sc-11351) was obtained from Santa Cruz Biotechnology, Inc. and anti-Bim antibodies were purchased from Stressgen or Chemicon International, Inc.

Cell culture, transfection, and Luc assay. 293T and Saos2 cells were grown in Dulbecco's modified Eagle's medium/F12 (Invitrogen) supplemented with 10\% fetal bovine serum (FBS). Transient transfections were performed with $1.6 \mu \mathrm{g}$ FRE-Luc or Fas-L-Luc as a reporter, and $0.4 \mu \mathrm{g}$ FOXO3a or an empty vector as an effector. The transfection agent, SN2 liposome or Lipofectamine 2000 (Invitrogen), was mixed with DNA in serum-free media and incubated for $30 \mathrm{~min}$. Cells were incubated with the transfection mix for $6 \mathrm{~h}$ before adding the FBS (10\%)-containing media. Cells were treated or untreated with IR (10 Gy or as indicated) $4 \mathrm{~h}$ before harvesting, and cell lysates for Luc activity were collected $24 \mathrm{~h}$ after transfection.
In all transfection experiments, the total amount of DNA was kept the same by addition of an empty vector. Plasmid pRL-tk (Promega) was used as an internal control in all luc transfection experiments. Error bars are the mean \pm standard error.

Western blot analysis. Specifically, $\sim 2 \times 10^{6}$ cells of Saos 2 cells were cultured for $16 \mathrm{~h}$ and treated or untreated with $\operatorname{IR}(5,10$, 15 Gy) after $30 \mathrm{~min}, 2,4,6,8,10$, and $24 \mathrm{~h}$. The protein samples were subjected to SDS-PAGE and transferred onto nitrocellulose membranes. The membranes were blocked with $5 \%$ non-fat dry milk in PBS containing $0.05 \%$ Tween-20 and incubated with primary antibodies (FOXO3a and Bim) and then with anti-rabbit peroxidase-conjugated secondary antibodies according to the manufacturer's instructions. The immunoblots were visualized using an enhanced chemiluminescence (ECL) kit obtained from Amersham Pharmacia Biotech.

In situ immunofluorescence staining. Specifically, $2 \times 10^{4}$ cells of Saos 2 were plated into chamber slides for $16 \mathrm{~h}$ and treated or untreated with 10 Gy IR after $1 \mathrm{~h}$. Cells were fixed in $4 \%$ paraformaldehyde for $1 \mathrm{~h}$ and permeabilization with $0.2 \%$ Triton X-100 for $30 \mathrm{~min}$, the cellular localization of FOXO3a was determined by using a polyclonal antibody against FOXO3a (1:200 dilution, Santa Cruz) followed by an FITCconjugated anti-rabbit IgG secondary antibody. After extensive washing in PBS, the samples were further incubated with DAPI $(0.1 \mathrm{~g} / \mathrm{ml})$ for $1 \mathrm{~h}$. After extensive washing, the samples were examined under a fluorescent microscope (Zeiss). Non-specific reaction of the secondary antibody was ruled out by the absence of fluorescence under the microscope.

Apoptosis assay. The apoptotic cells were analyzed by the terminal deoxynucleotidyltransferase (TdT)-mediated dUTP nick-end labeling (TUNEL) assay as described (26). Specifically, Saos 2 cells were treated with 10 Gy IR, incubated for $72 \mathrm{~h}$, harvested and collected by cytopreparations with a Cytospin-2 cytocentrifuge at a concentration of $\sim 3 \times 10^{3}$ cells/ slide. Cells were fixed with $4 \%$ formaldehyde for $10 \mathrm{~min}$, washed, and immersed in $0.5 \%$ Triton X-100 buffer for $15 \mathrm{~min}$. The fixed cells were treated with proteinase $\mathrm{K}(20 \mu \mathrm{g} / \mathrm{ml})$ for $15 \mathrm{~min}$ prior to the TUNEL assay. The slides were rinsed with distilled $\mathrm{H}_{2} \mathrm{O}$ and immersed in TDT buffer $(30 \mathrm{mM}$ Tris, $\mathrm{pH}$ 7.2, $140 \mathrm{mM}$ sodium cacodylate, $1 \mathrm{mM}$ cobalt chloride) for $15 \mathrm{~min}$. TdT $(0.3 \mathrm{U} / \mu \mathrm{l})$ and biotinylated dUTP $(20 \mathrm{mM})$ in TDT buffer were added to cover the cells on the slides, which were incubated at $37^{\circ} \mathrm{C}$ for $60 \mathrm{~min}$. The slides were transferred to TB buffer $(300 \mathrm{mM}$ sodium chloride, $30 \mathrm{mM}$ sodium citrate) at room temperature for $15 \mathrm{~min}$ to terminate the reaction, then covered with $10 \%$ normal horse serum for 30 min, washed, and stained with ABC (avidin biotin complex) at $37^{\circ} \mathrm{C}$ for $60 \mathrm{~min}$.

\section{Results}

Radiation enhances FOXO3a transcriptional activity. To address whether radiation induces FOXO3a transcriptional activity, we examined the effect of IR on the FOXO3adependent transcriptional activation of FRE-Luc (a luciferase reporter gene containing FOXO response elements upstream of a basic promoter). After co-transfecting FRE-Luc with a 
A

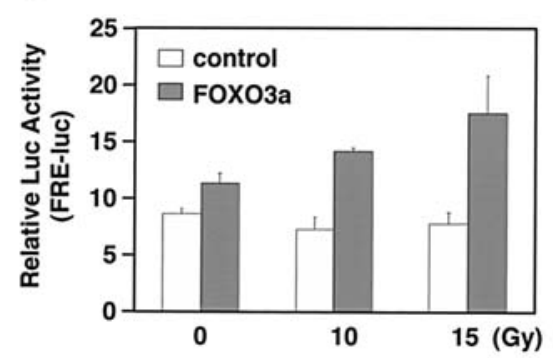

B

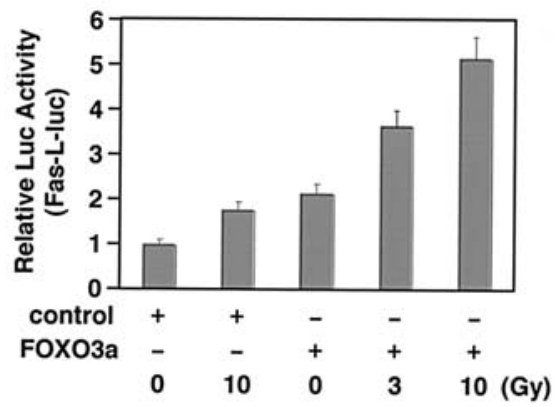

Figure 1. Ionizing radiation (IR) induces FOXO3a transcriptional activity. (A) Effect of IR stimulation on FOXO3a induced transactivation of FREluciferase activity. $293 \mathrm{~T}$ cells were co-transfected with control vector, FOXO3a and FRE-Luc after $24 \mathrm{~h}$ and then either untreated or treated with 10 and 15 Gy of ionizing radiation. After $4 \mathrm{~h}$, luciferase assays were performed. The results were collected by triple independent assays and each sample was duplicated. (B) $293 \mathrm{~T}$ cells were co-transfected with control vector, FOXO3a and Fas-L-Luc after $24 \mathrm{~h}$ and then either untreated or treated with 3 and $10 \mathrm{~Gy}$ of ionizing radiation. After $4 \mathrm{~h}$, luciferase assays were performed. (The results were collected by two independent assays and each sample was duplicated).

FOXO3a expression vector or control vector into 293T cells followed by IR treatment, we performed luciferase assays and showed that IR promoted the activity of FOXO3a to upregulate
FRE-Luc in cells (Fig. 1A). Similarly, UV treatment also significantly induced FOXO3a transcriptional activity in 293T cells co-transfected with FRE-Luc and FOXO3a (data not shown). To further address whether this phenomenon can be detected using a FOXO3a induced gene such as Fas-L, we co-transfected Fas-L-Luc (a luciferase reporter driven by the promoter of Fas-L) with a FOXO3a expression vector or control vector into $293 \mathrm{~T}$ cells followed by IR treatment and examined the response of Fas-L-Luc. Consistently, we showed that the luciferase activity of Fas-L-Luc was induced in a dose-dependant manner in response to IR (Fig. 1B), suggesting that IR activates the FOXO3a transcriptional activity.

Radiation increases $\mathrm{FOXO}$ a protein expression. To further demonstrate whether FOXO3a protein level was also induced in response to radiation, we determined the endogenous FOXO3a protein level by Western blot analysis in Saos2 cells after IR. We showed that the FOXO3a protein level was increased in a dose-dependant fashion in response to IR (Fig. 2A), and could be detected within 30 min after treatment with 10 Gy (Fig. 2B). A similar phenomenon was also detected in Saos 2 cells in response to UV treatment (data not shown), suggesting that radiation augments the FOXO3a protein expression in Saos 2 cells.

IR regulates $\mathrm{FOXO3a} \mathrm{subcellular} \mathrm{localization.} \mathrm{It} \mathrm{has} \mathrm{been}$ shown that cellular localization of FOXO3a between the nucleus and the cytoplasm is primarily regulated by certain serine/threonine protein kinases such as Akt $(6,7)$. However, it is unknown whether localization of FOXO3a can be modulated by genotoxic stress such as radiation. To determine whether IR regulates FOXO3a nuclear localization, we examined subcellular localization of FOXO3a in response to IR treatment by using immunofluorescence staining with an antibody specific to FOXO3a after IR. Our results showed
A
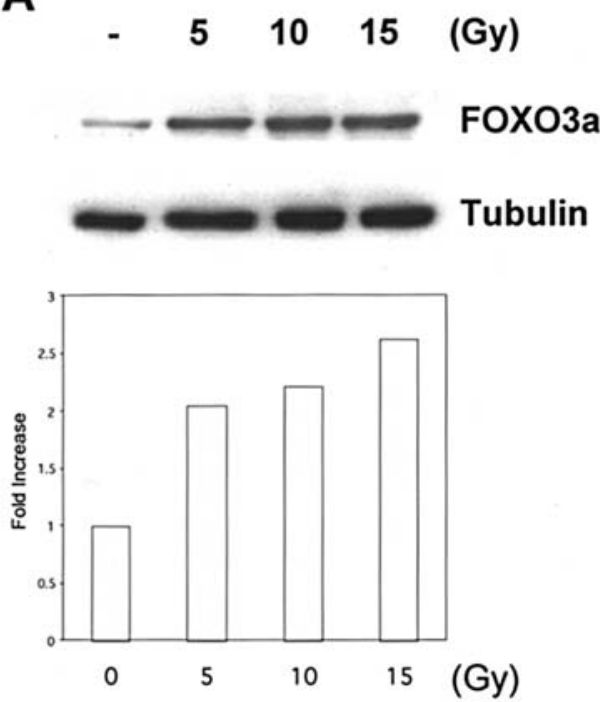

B
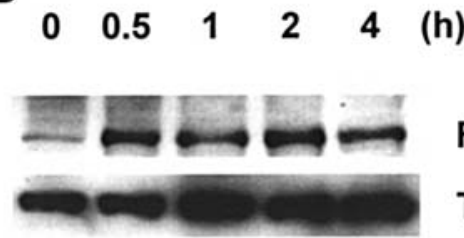

FOXO3a

Tubulin

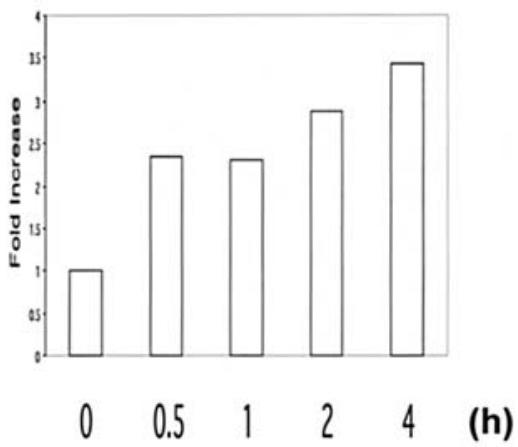

Figure 2. Ionizing radiation (IR) enhances FOXO3a protein expression. (A) Saos2 cells were either untreated or treated with 5 , 10 and 15 Gy of ionizing radiation for $4 \mathrm{~h}$ and then harvested. Western blot experiments were performed with antibodies to endogenous FOXO3a (top) and tubulin (bottom). (B) Saos2 cells were either untreated or treated with $10 \mathrm{~Gy}$ of ionizing radiation. After $30 \mathrm{~min}, 1,2$ and $4 \mathrm{~h}$, whole cell lysates were prepared and then Western blot experiments were performed with antibodies to endogenous FOXO3a (top) and tubulin (bottom). 
A FOXO3a

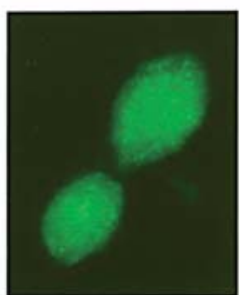

$10 \mathrm{~Gy}$

$0 \mathrm{~Gy}$

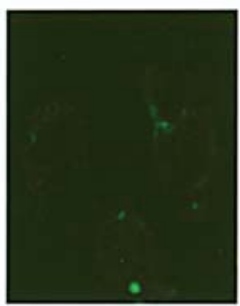

DAPI
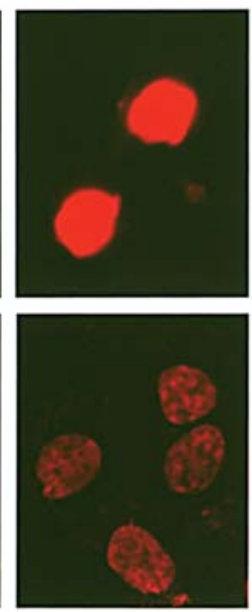

Merge
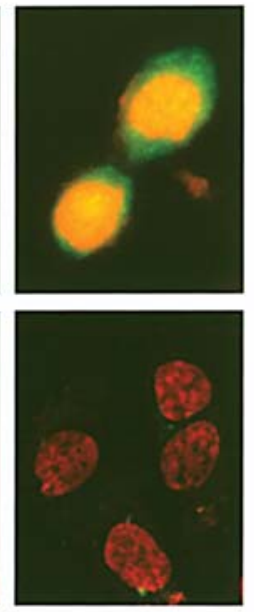

B

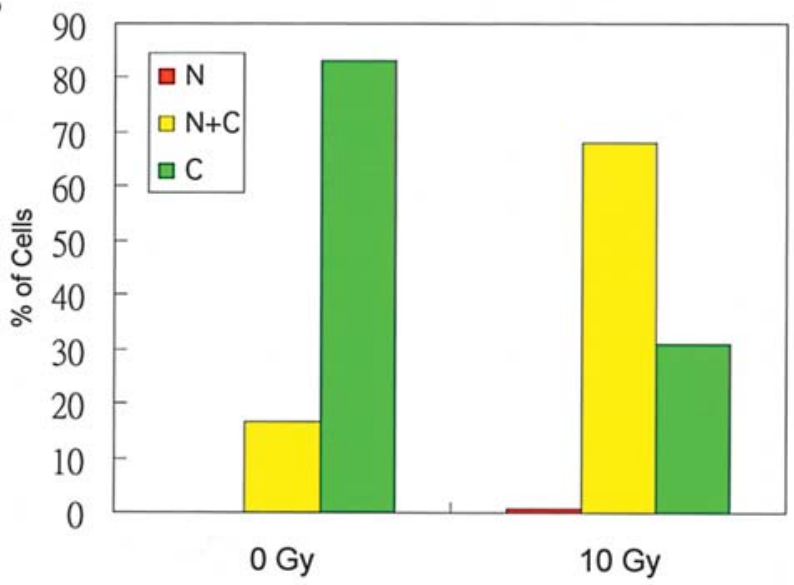

Figure 3. Ionizing radiation (IR) promotes FOXO3a nuclear localization. (A) Saos 2 cells were either untreated or treated with 10 Gy of IR for $1 \mathrm{~h}$. Subsequently, cells were fixed and the cellular localization of endogenous FOXO3a was detected using an anti-FOXO3a polyclonal antibody. After extensive washing in PBS, samples were further incubated with DAPI and examined by fluorescence microscopy. Each image represents 2 sections at the same cellular level and magnification. (B) After DNA co-transfection and IR treatment as shown, an average (\%) of 300-400 FOXO3a-positive cells in the nucleus $(\mathrm{N})$ or cytoplasm $(\mathrm{C})$ of untreated or treated cells was determined.

that, consistent with Fig. 2A, IR induced a high expression of FOXO3a that was predominantly localized in the nucleus in Saos 2 cells (Green) as compared to that in the untreated cells, where the low level expression of FOXO3a stayed primarily in the cytoplasm (Fig. 3A). We further verified this IR-induced nuclear localization of FOXO3a by examining an average of 300-400 FOXO3a-positive cells. Our results showed that FOXO3a was mainly detected in the nucleus $(\mathrm{N}$ and $\mathrm{N}+\mathrm{C})$ of $\sim 68 \%$ of the FOXO3a-positive cells treated with IR, whereas FOXO3a was found in the nucleus of $\sim 16 \%$ of the untreated FOXO3a-positive cells (Fig. 3B). Collectively, our results indicate that IR promotes FOXO3a nuclear localization in Saos 2 cells.

Radiation stimulates expression of Bim, a FOXO3a target gene, and induces cellular apoptosis. To further address whether

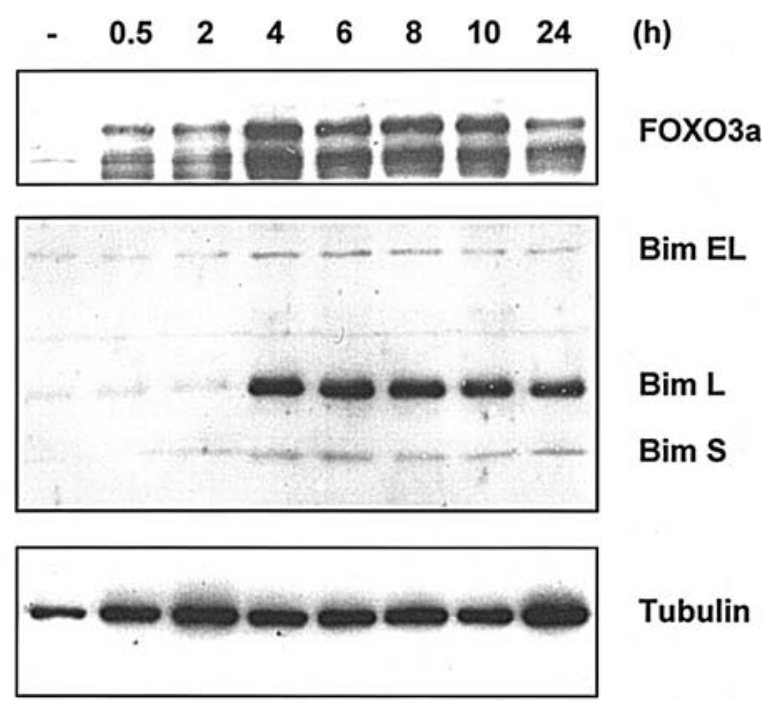

Figure 4. Ionizing radiation (IR) stimulates Bim protein expression and apoptosis. Saos 2 cells were either untreated or treated with 10 Gy of ionizing radiation. After incubation for $0.5,2,4,6,8,10$, and $24 \mathrm{~h}$ as indicated, whole cell lysates were prepared and then Western blot experiments were performed with specific antibodies against FOXO3a (top panel) or Bim (central panel) or tubulin (bottom panel) as a loading control.

A

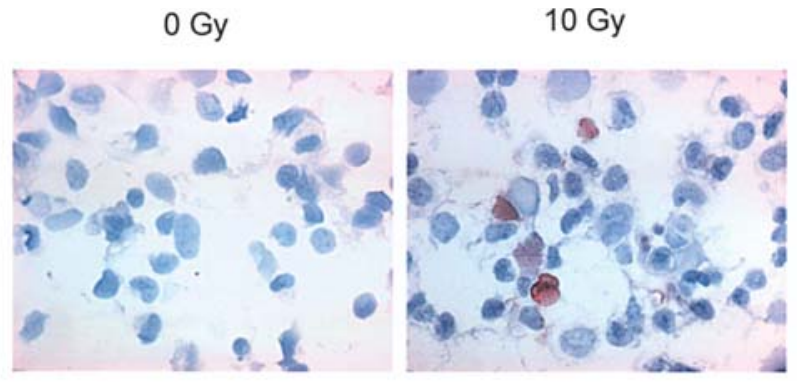

B

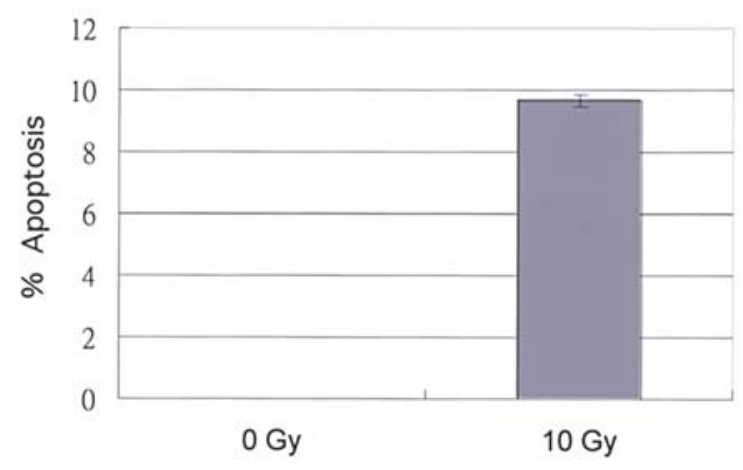

Figure 5. Ionizing radiation (IR) induces cellular apoptosis. Saos2 cells were either untreated ( 0 Gy) or treated with (10 Gy) IR. (A) After incubation for $72 \mathrm{~h}$, the cells were collected on the slides by cytopreparations with a Cytospin-2 cytocentrifuge for TUNEL assays. (B) An average (\%) of apoptotic (TUNEL-positive) cells in three independent sections on slides was determined (bottom panel).

FOXO3a induced endogenous gene expression in vivo, we analyzed Bim protein expression in Saos 2 cells after IR treatment by Western blot analysis since Bim has been shown to be regulated by FOXO3a previously $(19,20)$. We showed that the endogenous FOXO3a protein level was enhanced within 


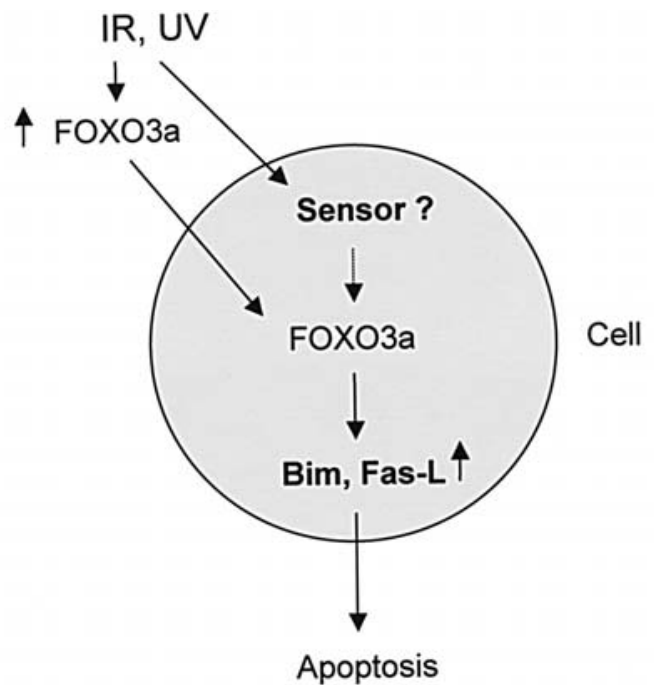

Figure 6. The model for FOXO3a-mediated apoptosis in response to genotoxic-stress such as IR or UV irradiation.

30 min of IR treatment (Fig. 4), which was consistent with the previous results (Fig. 2B). Moreover, the endogenous Bim protein expression was also increased in Saos 2 cells after $4 \mathrm{~h}$ of IR treatment (Fig. 4). Similar phenomena were also detected in Saos2 cells in response to UV treatment (data not shown), indicating that radiation upregulates expression of FOXO3a and its target, Bim, in Saos 2 cells. To validate whether IR treatment under the same condition (10 Gy) induced cellular apoptosis, we analyzed Saos 2 cells treated or untreated (control) with IR by TUNEL assays. As shown in Fig. 5, $\sim 10 \%$ of apoptotic cells were evidently identified in the cells treated with IR but not in the control cells, suggesting that upregulation of Bim and FOXO3a correlates with cell apoptosis induced by IR.

\section{Discussion}

Upregulation and nuclear localization (activation) of the FOXO transcription factors induced by genotoxic stress have not been reported, and the roles of FOXO transcription factors in genotoxic stress-mediated apoptosis have not been determined. Our results provide evidence that the FOXO3a protein level is increased and FOXO3a translocates into the nucleus and activates its target genes, Fas- $\mathrm{L}$ and Bim, in response to radiation. Since this phenomenon is discovered in a p53-null cell line, Saos2, it suggests that upregulation and activation of FOXO3a and Bim by radiation may be through a p53independent pathway. It is known that the tumor suppressor, p53, plays a key role in genotoxic stress responses, including repair of DNA damage, cell-cycle arrest, and apoptosis, which is complicated and mostly through a p53-dependent pathway (1-4). Thus, we propose a new mechanism by which FOXO3a induces apoptosis in response to radiation through upregulation of Fas-L or Bim in a p53-independent manner (Fig. 6).

In proliferating cells, the binding of growth factors to their specific receptor tyrosine kinases activates the phosphoinositide 3-kinase (PI3K)/Akt signaling pathway that in part induces directly phosphorylating and inhibiting members of the FOXO subfamily of forkhead transcription factors $(18,25,27)$. How- ever, in the absence of PI3K/Akt signaling, FOXO transcription factors and their cellular target genes are activated $(18,25,27)$. In most cell types, this leads to cell-cycle arrest and quiescence but not apoptosis (14-16). For instance, in the nematode Caenorhabditis elegans, an absence of Akt signaling leads to activation of the worm FOXO transcription factor, DAF-16, resulting in either extension of the adult life-span or entrance into the long-lived larval stage termed dauer $(28,29)$. Studies in mammalian cells have shown that ectopic expression of FOXO1, FOXO3a, and FOXO4 can induce either cell-cycle arrest or apoptosis $(6,14,19,30)$.

It has been well documented that nuclear localization of FOXO3a is negatively regulated by Akt or Akt-like serine/ threonine protein kinases in cell survival signaling pathways (6-10). In agreement with the survival mechanisms, our findings indicate that nuclear localization of FOXO3a is positively modulated by genotoxic stress such as IR or UV irradiation that can induce cell apoptosis. The complicated and coordinated regulations of FOXO transcription factors in response to a variety of environmental stimuli suggest that FOXO transcription factors may play a versatile and important role in regulating critical cellular functions such as cell-cycle arrest, irreversible senescence, and apoptosis, probably depending on the physiological or pathological conditions and/or cell types.

Further elucidation of the molecular mechanisms underlying DNA damage and genomic instability induced by radiation may provide new targets that can be exploited therapeutically. Further investigation of the signaling mechanism by which radiation activates FOXO3a that in turn contributes to apoptosis is necessary for understanding the molecular basis for radiationinduced apoptosis and/or application as an anti-cancer radiation therapy.

\section{Acknowledgments}

We gratefully acknowledge Drs K.C. Arden, M.E. Greenberg, and C.V. Paya for providing critical reagents, and Dr M.-C. Hung for his encouragement and excellent support. This work was carried out in part in the group of Dr M.-C. Hung, and supported in part by grants from the Susan G. Komen Breast Cancer Foundation (BCTR0201848 and BCTR0504415, M.H.), the start-up funds from MDACC (M.H. and M.C.H.), NIH (1R01 CA113859-01A1 M.H. and CA58880 M.C.H.), PO1 (CA99031 M.C.H.), and Cancer Center Supporting Grant CA16772.

\section{References}

1. Little JB: Genomic instability and radiation. J Radiol Prot 23: 173-181, 2003.

2. Huang L, Snyder AR and Morgan WF: Radiation-induced genomic instability and its implications for radiation carcinogenesis. Oncogene 22: 5848-5854, 2004.

3. Pluquet $\mathrm{O}$ and Hainaut P: Genotoxic and non-genotoxic pathways of p53 induction. Cancer Lett 174: 1-15, 2001.

4. Gudkov AV and Komarova EA: The role of p53 in determining sensitivity to radiotherapy. Nat Rev Cancer 3: 117-129, 2003.

5. Prise KM, Schettino G, Folkard M and Held KD: New insights on cell death from radiation exposure. Lancet Oncol 6: 520-528, 2005.

6. Burgering BM and Kops GJ: Cell cycle and death control: long live Forkheads. Trends Biochem Sci 27: 352-360, 2002.

7. Tran H, Brunet A, Griffith EC and Greenberg ME: The Many Forks in FOXO's Road. Sci STKE 172: re5, 2003. 
8. Accili D and Arden KC: FoxOs at the crossroads of cellular metabolism, differentiation, and transformation. Cell 117: 421-426, 2004.

9. Finnberg $\mathrm{N}$ and El-Deiry WS: Activating FOXO3a, NF-кB and p53 by targeting IKKs: an effective multi-faceted targeting of the tumor-cell phenotype? Cancer Biol Ther 3: 614-616, 2004.

10. Coffer PJ and Burgering BM: Forkhead-box transcription factors and their role in the immune system. Nat Rev Immunol 4: 889-899, 2004

11. Barthel A, Schmoll D and Unterman TG: FoxO proteins in insulin action and metabolism. Trends Endocrinol Metab 16: 183-189, 2005.

12. Furukawa-Hibi Y, Kobayashi Y, Chen C and Motoyama N: FOXO transcription factors in cell-cycle regulation and the response to oxidative stress. Antioxid Redox Signal 7: 752-760, 2005.

13. Carlsson P and Mahlapuu M: Forkhead transcription factors: key players in development and metabolism. Dev Biol 250: $1-23,2002$.

14. Medema RH, Kops GJ, Bos JL and Burgering BM: AFX-like Forkhead transcription factors mediate cell-cycle regulation by Ras and PKB through p27kip1. Nature 404: 782-787, 2000.

15. Kops GJ, Dansen TB, Polderman PE, Saarloos I, Wirtz KW, Coffer PJ, Huang TT, Bos JL, Medema RH and Burgering BM: Forkhead transcription factor FOXO3a protects quiescent cells from oxidative stress. Nature 419: 316-321, 2002.

16. Kops GJ, Medema RH, Glassford J, Essers MA, Dijkers PF, Coffer PJ, Lam EW and Burgering BM: Control of cell cycle exit and entry by protein kinase B-regulated forkhead transcription factors. Mol Cell Biol 22: 2025-2036, 2002.

17. Alvarez B, Martinez AC, Burgering BM and Carrera AC: Forkhead transcription factors contribute to execution of the mitotic programme in mammals. Nature 413: 744-747, 2001.

18. Brunet A, Bonni A, Zigmond MJ, Lin MZ, Juo P, Hu LS, Anderson MJ, Arden KC, Blenis J and Greenberg ME: Akt promotes cell survival by phosphorylating and inhibiting a Forkhead transcription factor. Cell 96: 857-868, 1999.

19. Dijkers PF, Birkenkamp KU, Lam EW, Thomas NS, Lammers JW, Koenderman L and Coffer PJ: FKHR-L1 can act as a critical effector of cell death induced by cytokine withdrawal: protein kinase B-enhanced cell survival through maintenance of mitochondrial integrity. J Cell Biol 156: 531-542, 2002.
20. Stahl M, Dijkers PF, Kops GJ, Lens SM, Coffer PJ, Burgering BM and Medema RH: The forkhead transcription factor FoxO regulates transcription of $\mathrm{p} 27 \mathrm{Kip} 1$ and Bim in response to IL-2. J Immunol 168: 5024-5031, 2002.

21. Modur V, Nagarajan R, Evers BM and Milbrandt J: FOXO proteins regulate tumor necrosis factor-related apoptosis inducing ligand expression. Implications for PTEN mutation in prostate cancer. J Biol Chem 277: 47928-47937, 2002.

22. Daly C, Wong V, Burova E, Wei Y, Zabski S, Griffiths J, Lai KM, Lin HC, Ioffe E, Yancopoulos GD and Rudge JS: Angiopoietin-1 modulates endothelial cell function and gene expression via the transcription factor FKHR (FOXO1). Genes Dev 18: 1060-1071, 2004.

23. Hu M C-T, Lee D-F, Xia W, Golman L, Ou-Yang F, Yang J-Y, Zou Y, Bao S, Hanada N, Saso H, Kobayashi R and Hung MC: IкB kinase promotes tumourigenesis through inhibition of Forkhead FOXO3a. Cell 117: 225-237, 2004.

24. Tran H, Brunet A, Grenier JM, Datta SR, Fornace AJ Jr, Di Stefano PS, Chiang LW and Greenberg ME: DNA repair pathway stimulated by the forkhead transcription factor FOXO3a through the Gadd45 protein. Science 296: 530-534, 2002.

25. Biggs WH III, Meisenhelder J, Hunter T, Cavenee WK and Arden KC: Protein kinase B/Akt-mediated phosphorylation promotes nuclear exclusion of the winged helix transcription factor FKHR1. Proc Natl Acad Sci USA 96: 7421-7426, 1999.

26. Deng J, Xia W and Hung MC: Adenovirus 5 E1A-mediated tumor suppression associated with E1A-mediated apoptosis in vivo. Oncogene 17: 2167-2175, 1998.

27. Guo S, Rena G, Cichy S, He X, Cohen P and Unterman T: Phosphorylation of serine 256 by protein kinase B disrupts transactivation by FKHR and mediates effects of insulin on insulin-like growth factor-binding protein-1 promoter activity through a conserved insulin response sequence. J Biol Chem 274: 17184-17192, 1999.

28. Lee SS, Kennedy S, Tolonen AC and Ruvkun G: DAF-16 target genes that control C. elegans life-span and metabolism. Science 300: 644-647, 2003.

29. Ogg S, Paradis S, Gottlieb S, Patterson GI, Lee L, Tissenbaum HA and Ruvkun G: The Fork head transcription factor DAF-16 transduces insulin-like metabolic and longevity signals in $\mathrm{C}$. elegans. Nature 389: 994-999, 1997.

30. Brunet A, Park J, Tran H, Hu LS, Hemmings BA and Greenberg ME: Protein kinase SGK mediates survival signals by phosphorylating the forkhead transcription factor FKHRL1 (FOXO3a). Mol Cell Biol 21: 952-965, 2001. 